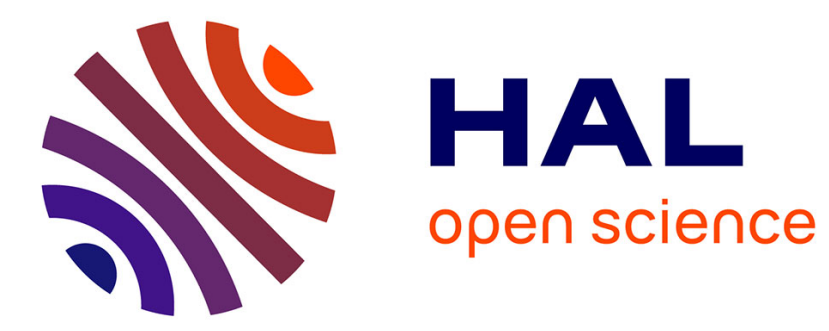

\title{
Snap-through truss as an absorber of forced oscillations
}

Konstantin Avramov, Yuri Mikhlin

\section{To cite this version:}

Konstantin Avramov, Yuri Mikhlin. Snap-through truss as an absorber of forced oscillations. Journal of Sound and Vibration, 2006, 290 (3-5), pp.705-722. 10.1016/j.jsv.2005.04.022 . hal-01347936

\section{HAL Id: hal-01347936 \\ https://hal.science/hal-01347936}

Submitted on 22 Jul 2016

HAL is a multi-disciplinary open access archive for the deposit and dissemination of scientific research documents, whether they are published or not. The documents may come from teaching and research institutions in France or abroad, or from public or private research centers.
L'archive ouverte pluridisciplinaire HAL, est destinée au dépôt et à la diffusion de documents scientifiques de niveau recherche, publiés ou non, émanant des établissements d'enseignement et de recherche français ou étrangers, des laboratoires publics ou privés.

\section{(c)(1)}

Distributed under a Creative Commons Attribution| 4.0 International License 


\title{
Snap-through truss as an absorber of forced oscillations
}

\author{
K.V. Avramov ${ }^{\mathrm{a}, *}$, Y.V. Mikhlin ${ }^{\mathrm{b}}$ \\ ${ }^{a}$ Department of Fluid and Gas Mechanics, Faculty of Physics and Engineering, National Technical University \\ "Kharkov Polytechnic Institute", Frunze St. 21, Kharkov 61002, Ukraine \\ bepartment of Applied Mathematics, National Technical University "Kharkov Polytechnic Institute", \\ Frunze St. 21, Kharkov 61002, Ukraine
}

\begin{abstract}
The possibility of absorption of linear elastic system forced oscillations by means of an essentially nonlinear absorber (snap-through truss), which is attached to the linear subsystem, is analyzed. The simplest mass-spring linear subsystem is chosen to study the problem of forced oscillations absorption. The combination of the nonlinear normal vibrations modes method, the Rauscher approach and the asymptotic analysis is used to study forced oscillations of the two-dof system. The absorption vibrations mode in the form of nonlinear normal vibrations mode with small oscillations amplitudes of the linear subsystem and large amplitudes of the snap-through truss is studied. It is shown that this mode is stable over the wide range of the excitation frequency.
\end{abstract}

\section{Introduction}

Many scientific publications contain description and analysis of different devices for passive and active absorption of mechanical oscillations. The theory of linear passive absorbers is considered in Ref. [1]. The basis of a passive absorption is energy pumping from a main elastic subsystem into an absorber. Note that the energy pumping in nonlinear systems is considered in 
Ref. [2]. Energy pumping between vibrations modes of geometrically nonlinear cylindrical shells is treated in Ref. [3].

Many efforts have been made to study vibrations absorption. A beam as an absorber, which is attached to a linear mass-spring system, is considered in Ref. [4]. The pendulum torsional vibration absorber is studied by Shaw and Wiggins [5]. They use the Melnikov method to study chaotic vibrations. If the nonlinear absorber is attached to the main linear subsystem, the unstable periodic oscillations take place close to the combination resonance and stable almost periodic stable oscillations occur [6]. Natsiavas [7] suggested to use a system with a nonlinear spring to absorb the forced oscillations of the Duffing system. The mass-spring nonlinear system is used to absorb oscillations of a self-excited subsystem [8]. Lee and Shaw [9] considered a quenching of torsional oscillations of the four-stoke, four-cylinder internal combustion engine by the centrifugal pendulum absorber. Haddow and Shaw [10] experimentally studied the rotating machinery with the centrifugal pendulum absorber, which is used in aircrafts and automobiles. The essentially nonlinear spring is considered as an absorber of free oscillations of the discrete mechanical system in Ref. [11]. The method of nonlinear integral equations is used to analyze this discrete system. A semi-infinite linear chain with an essentially nonlinear absorber is considered in Ref. [12]. A single-dof main elastic system with the absorber, which consists of a set of pendulums, is treated in Ref. [13].

Impact systems are used to absorb oscillations of discrete and continuous mechanical systems [14,15]. Aoki and Watanabe [16] studied the impact absorber (the small mass hitting on stops). Shaw and Shaw [17] suggested a reverse pendulum hitting on stops to absorb oscillations of a single-dof subsystem.

In the present paper a simple model of an elastic system (a single-dof mass-spring oscillator) under the time-periodic force is used to study a possibility of absorption by means of the snapthrough truss. The mass and stiffness of the absorber are smaller, than the corresponding parameters of the main linear subsystem, which is the principal design condition. The vibration absorption mode with significant vibrations amplitudes of the snap-through truss and small amplitudes of the main linear subsystem is treated. The combination of the nonlinear normal vibrations mode method, the Rauscher approach and asymptotic analysis is used to study vibration absorption mode.

\section{Equations of motions}

Fig. 1 shows the system under consideration. The motions under the action of time-periodic force $F \cos \Omega t$ are defined by two generalized coordinates $U, W$. These coordinates $W, U$ are counted off from the stable equilibrium position. The equations of motions are derived with respect to the dimensionless variables and parameters:

$$
(u, w)=(U, W) L^{-1}, \quad \tau=\omega_{0} t, \quad \omega_{0}^{2}=\frac{\tilde{c}_{1}}{M}, \quad \omega=\frac{\Omega}{\omega_{0}}, \quad \gamma=\frac{\tilde{c}}{\tilde{c}_{1}}, \quad \mu=\frac{m}{M}, \quad \varepsilon f=\frac{F}{\tilde{c}_{1} L} \quad(\varepsilon \ll 1),
$$

where $M, m$ are masses of the main linear subsystem and the snap-through absorber; $L, \tilde{c}$ are length and stiffness of the spring; $(u, w)$ are the dimensionless generalized coordinates; $\tau$ is the 


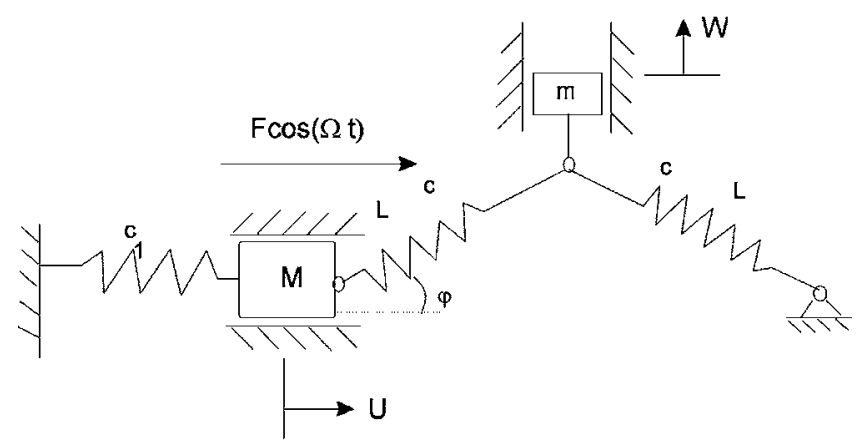

Fig. 1. The mechanical system under consideration.

dimensionless time; $\omega_{0}^{2}$ is the partial frequency of the linear subsystem; $\omega$ is the dimensionless frequency of the external excitation. The equations of system motions are

$$
\begin{gathered}
\ddot{u}+\beta_{1} \dot{u}+u-\gamma(c-u)\left(1-\frac{1}{\sqrt{(s+w)^{2}+(c-u)^{2}}}\right)=\varepsilon f \cos (\omega \tau), \\
\mu \ddot{w}+\beta_{2} \dot{w}+\gamma(s+w)\left(2-\frac{1}{\sqrt{(s+w)^{2}+c^{2}}}-\frac{1}{\sqrt{(s+w)^{2}+(c-u)^{2}}}\right)=0,
\end{gathered}
$$

where $c=\cos \varphi ; s=\sin \varphi$. Angle $\varphi$ defines the stable equilibrium position of the snap-through absorber (Fig. 1). We assume, that damping forces $\beta_{1} \dot{u}$ and $\beta_{2} \dot{w}$ act on both masses of the system. However, damping is not taken into account in the analytical analysis, which is presented below. The damping forces are considered only in the numerical simulations of system (2).

Note that in the previous paper of the authors [18] it is analyzed the snap-through motions which exist without any external force. The snap-through forced oscillations take place at an arbitrary value of $f$, which is confirmed by the numerical simulations. Different kinds of forced oscillations of system (2) can be observed besides the snap-through motions, which are considered here. The motions, when the snap-through truss performs small oscillations close to the stable equilibrium position, are considered in the previous paper of the authors [19].

Note that the snap-through truss is parametrically excited by the main mass motions (Fig. 1). In this case the complex bifurcation behavior of the absorber and chaotic vibrations could take place. The first results devoted to this subject are published in Ref. [20].

As the stiffness and mass of the snap-through absorber are significantly smaller than the corresponding parameters of the main linear subsystem, we assume that

$$
\gamma=\varepsilon \bar{\gamma}, \quad \mu=\varepsilon \bar{\mu} .
$$

New generalized coordinates $w_{1}, u_{1}$, which are counted off from the unstable equilibrium position, are introduced in the following form:

$$
w_{1}=w+s, \quad u_{1}=u-u_{*},
$$


where $u_{*}=\gamma(c-1) /(1+\gamma)$. System (2) is rewritten with respect to $\left(u_{1}, w_{1}\right)$ and the Taylor-series expansions are performed, retaining the terms up to the third order. As a result the following system of the differential equations is derived:

$$
\begin{gathered}
\ddot{u}_{1}+u_{1}=\varepsilon \bar{\gamma}\left\{-u_{1}+\frac{15}{4} c s^{2} u_{1}^{2}+c\left(\frac{1}{2}+\frac{3}{4} s^{2}\right) w_{1}^{2}+\left(1-\frac{9}{4} s^{2}\right) u_{1} w_{1}^{2}+\left(\frac{5}{2}-\frac{15}{4} s^{2}\right) u_{1}^{3}\right\}+\varepsilon f \cos \omega \tau, \\
\ddot{w}_{1}+p^{2} w_{1}\left[-s^{2}\left(1+\frac{3}{4} s^{2}\right)-c\left(1+\frac{3}{2} s^{2}\right) u_{1}-\left(1-\frac{9}{4} s^{2}\right) u_{1}^{2}+\left(1+\frac{3}{2} s^{2}\right) w_{1}^{2}\right]=0 .
\end{gathered}
$$

Note that the corresponding unperturbed $(\varepsilon=0)$ equations of system (5) are essentially nonlinear.

The motions of the main linear subsystem auto parametrically excite the snap-through absorber. But the motions of this system may excite the absorber directly, if the absorber dynamics is described by two general coordinates, that is the horizontal oscillations of absorber are taken into account too. In this case three-dof system has to be considered.

\section{Vibrations absorption mode}

The vibrations absorption mode is considered in the form of nonlinear normal vibrations mode (NNM) [21,22]. These motions have significant oscillations amplitudes of the snap-through absorber and small amplitudes of the main linear subsystem. The next estimation of the vibration absorption mode follows from Eqs. (5): $u_{1}=O(\varepsilon) ; w_{1}=O(1)$. Therefore, the corresponding zero approximation $(\varepsilon=0)$ can be presented as $u_{1}=0 ; w_{1}=O(1)$. Then variable $w_{1}$ of the zero approximation satisfies the differential equation:

$$
\ddot{w}_{1}+p^{2} w_{1}\left[-s^{2}\left(1+\frac{3}{4} s^{2}\right)+\left(1+\frac{3}{2} s^{2}\right) w_{1}^{2}\right]=0 .
$$

Eq. (6) describes two kinds of periodic motions, which are separated by homoclinic orbits (Fig. 2). Periodic orbit $L_{1}$ corresponds to a snap-through motion. As system (6) is conservative, solutions of this equation can be derived using the energy integral and the separation of variables. Details of

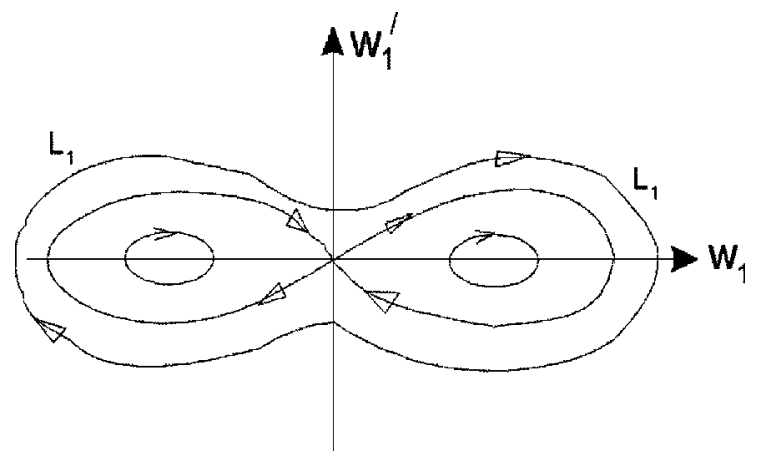

Fig. 2. The qualitative phase plane of Eq. (6). 
such equations analysis can be found in Ref. [23]. The solutions of Eq. (6) are:

$$
\begin{gathered}
\frac{p}{\sqrt{2}}\left(1+\frac{3}{4} s^{2}\right) \tau=-\int_{W_{1}^{(\max )}}^{w_{1}} \frac{\mathrm{d} w}{\sqrt{\left(W_{1}^{(\max )^{2}}-w^{2}\right)\left(w^{2}+b^{2}\right)}}, \\
W_{1}^{(\max )^{2}}=\left(s^{2}+\sqrt{s^{4}+4 H p^{-2}}\right)\left(1-\frac{3}{4} s^{2}\right), \\
b^{2}=\left(\sqrt{s^{4}+4 H p^{-2}}-s^{2}\right)\left(1-\frac{3}{4} s^{2}\right),
\end{gathered}
$$

where $H$ is a total energy of oscillator (6); $W_{1}^{(\max )}$ is the oscillations amplitude. Solution (7) can be rewritten as

$$
p \tau=\frac{\sqrt{1-\frac{3}{4} s^{2}}}{\left(s^{4}+4 H p^{-2}\right)^{1 / 4}} F(\varphi, k),
$$

where $F(\varphi, k)$ is the elliptic integral; $k$ is a modulus of the elliptic integral. The following equation connects $k$ to $W_{1}^{(\max )}$ and $H$ :

$$
k^{2}=\frac{\sqrt{s^{4}+4 H p^{-2}}+s^{2}}{2 \sqrt{s^{4}+4 H p^{-2}}}=\frac{1}{2\left(1-s^{2} W_{1}^{(\max )^{-2}}\right)} .
$$

Eqs. (9) are derived from Eq. (7). As motions of the snap-through absorber are considered outside the homoclinic trajectory, then $W_{1}^{(\max )}>s \sqrt{2}$ and $k \in\left[1 ; \frac{1}{\sqrt{2}}\right]$.

Solutions of Eq. (6) can be presented as

$$
w_{1}=W_{1}^{(\max )} \cos \varphi .
$$

Using the principal concept of Rauscher method [21,22], the zero approximation of solutions (8) are substituted into system (5). Precisely, solution (8) is substituted only into term $\varepsilon f \cos \omega \tau$ :

$$
\varepsilon f g(\varphi)=\varepsilon f g\left(w_{1}\right)=\varepsilon f \cos \left(\frac{\omega \sqrt{\left(1-\frac{3}{4} s^{2}\right)\left(2 k^{2}-1\right)}}{p s} F(\varphi, k)\right) .
$$

Frequency $\omega$ is extracted from the periodicity condition $g(\varphi)=g(\varphi+2 \pi)$ and Eq. (11):

$$
\omega=\frac{\pi s p}{2 K(k) \sqrt{\left(1-\frac{3}{4} s^{2}\right)\left(2 k^{2}-1\right)}},
$$

where $K(k)$ is the complete elliptic integral of the first kind. Then excitation (11) can be presented as

$$
\varepsilon f g(\varphi)=\varepsilon f \cos \left[\frac{\pi}{2 K(k)} F(\varphi, k)\right] .
$$

Moreover, the equation: $g(\pi+\varphi)=-g(\varphi)$ is satisfied. Therefore, we approximate the function $g(\varphi)$ by two harmonics of the Fourier series:

$$
g(\varphi) \approx A_{1} \cos \varphi+A_{3} \cos 3 \varphi+\cdots,
$$


where

$$
A_{n}=\frac{4 K(k)}{\pi^{2}} \int_{0}^{\pi} \cos \psi \cos \left[n a m\left(\frac{2 K(k)}{\pi} \psi\right)\right] \mathrm{d} n\left(\frac{2 K(k)}{\pi} \psi\right) \mathrm{d} \psi, \quad n=1,3, \operatorname{am}\left(\frac{2 K(k)}{\pi} \psi\right)
$$

is the Jacobi amplitude; $\mathrm{d} n(2 K \psi / \pi)$ is the Jacobi function. Using the Fourier series for $\operatorname{sn}(2 K \psi / \pi)$ [24], we derived:

$$
\begin{gathered}
A_{1}=\frac{2}{\pi} \int_{0}^{\pi} \sin \psi \operatorname{sn}\left(\frac{2 K \psi}{\pi}\right) \mathrm{d} \psi=\frac{\pi}{k K(k) \operatorname{sn}\left(\pi K^{\prime} / 2 K\right)}, \\
A_{3}=\frac{2}{\pi} \int_{0}^{\pi} \operatorname{sn}\left(\frac{2 K \psi}{\pi}\right) \sin \psi \mathrm{d} \psi-\frac{8}{3 \pi} \int_{0}^{\pi} \operatorname{sn}^{3}\left(\frac{2 K \psi}{\pi}\right) \sin \psi \mathrm{d} \psi .
\end{gathered}
$$

Using the Fourier series for $\operatorname{sn}^{3}(2 K \psi / \pi)$, which is presented in Appendix 2, the following equation is obtained:

$$
A_{3}=\frac{\pi\left[2 K^{2}\left(k^{2}-2\right)+\pi^{2}\right]}{6 k^{3} K^{3} \operatorname{sn}\left(\pi K^{\prime} / 2 K\right)} .
$$

Thus amplitudes of harmonics $A_{1}, A_{3}$ are obtained analytically.

Expression (11) is substituted into Eq. (5). Then the autonomous dynamical system is derived:

$$
\begin{gathered}
\ddot{u}_{1}+u_{1}=\varepsilon \bar{\gamma}\left\{-u_{1}+\frac{15}{4} c s^{2} u_{1}^{2}+c\left(\frac{1}{2}+\frac{3}{4} s^{2}\right) w_{1}^{2}+\left(1-\frac{9}{4} s^{2}\right) u_{1} w_{1}^{2}+\left(\frac{5}{2}-\frac{15}{4} s^{2}\right) u_{1}^{3}+f g\left(w_{1}\right)\right\}, \\
\ddot{w}_{1}+p^{2} w_{1}\left[-s^{2}\left(1+\frac{3}{4} s^{2}\right)-c\left(1+\frac{3}{2} s^{2}\right) u_{1}-\left(1-\frac{9}{4} s^{2}\right) u_{1}^{2}+\left(1+\frac{3}{2} s^{2}\right) w_{1}^{2}\right]=0 .
\end{gathered}
$$

We consider the absorption motions of system (18) in the form of nonlinear normal vibrations mode $u_{1}\left(w_{1}\right)$. Fig. 3 shows a qualitative trajectory of such motion. The NNM can be presented

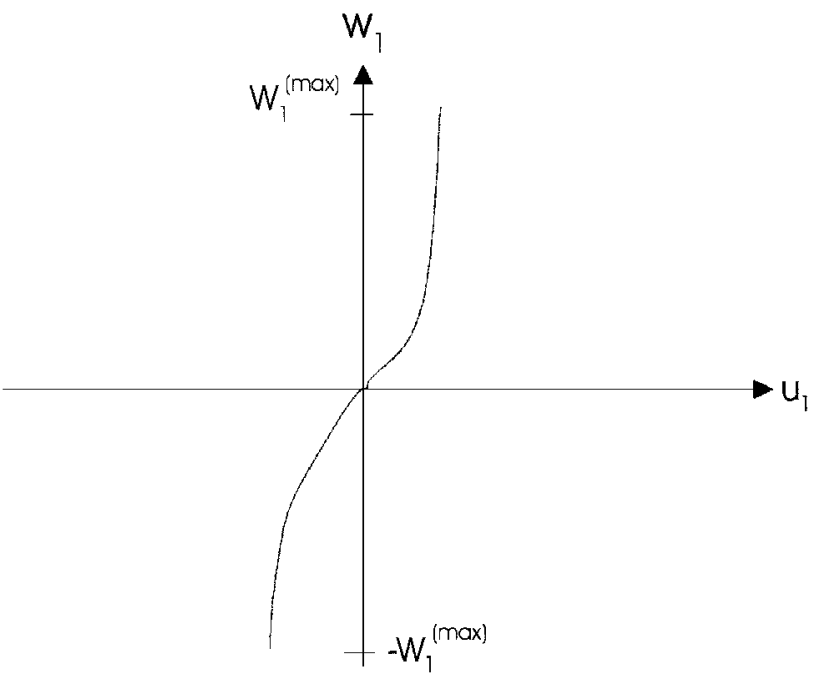

Fig. 3. Qualitative representation of the nonlinear normal mode in a configuration space. 
as $[21,22]$

$$
u_{1}=\varepsilon \tilde{u}\left(w_{1}\right)=\varepsilon\left(a_{0}+a_{1} w_{1}+a_{2} w_{1}^{2}+\cdots\right),
$$

where $a_{0}, a_{1}, a_{2}, \ldots$ are unknown constants. Using relation

$$
\frac{\mathrm{d}^{2} u_{1}}{\mathrm{~d} \tau^{2}}=\frac{\mathrm{d}^{2} u_{1}}{\mathrm{~d} w_{1}^{2}} \dot{w}_{1}^{2}+\frac{\mathrm{d} u_{1}}{\mathrm{~d} w_{1}} \ddot{w}_{1},
$$

Eq. (18a) can be presented as

$$
\begin{aligned}
& u_{1}^{\prime \prime} \dot{w}_{1}^{2}-p^{2}\left[-s^{2}\left(1+\frac{3}{4} s^{2}\right) w_{1}-c\left(1+\frac{3}{2} s^{2}\right) w_{1} u_{1}-\left(1-\frac{9}{4} s^{2}\right) u_{1}^{2} w_{1}+\left(1+\frac{3}{2} s^{2}\right) w_{1}^{3}\right] u_{1}^{\prime}+u_{1} \\
& \quad=\varepsilon \bar{\gamma}\left\{\left\{-u_{1}+\frac{15}{4} c s^{2} u_{1}^{2}+c\left(\frac{1}{2}+\frac{3}{4} s^{2}\right\}\right) w_{1}^{2}+\left(1-\frac{9}{4} s^{2}\right) u_{1} w_{1}^{2}+\left(\frac{5}{2}-\frac{15}{4} s^{2}\right) u_{1}^{3}\right\}+\varepsilon f g\left(w_{1}\right),
\end{aligned}
$$

where ()$^{\prime}=\mathrm{d}() / \mathrm{d} w_{1}$. The following equation is obtained from Eq. (18b):

$$
\begin{aligned}
& \frac{\dot{w}_{1}^{2}}{2}+p^{2} \int_{W_{1}^{(\max )}}^{w_{1}}\left[-s^{2}\left(1+\frac{3}{4} s^{2}\right) w_{1}+\left(1+\frac{3}{2} s^{2}\right) w_{1}^{3}\right] \mathrm{d} w_{1} \\
& \quad+p^{2} \int_{W_{1}^{(\max )}}^{w_{1}} w_{1}\left[-c\left(1+\frac{3}{2} s^{2}\right) u_{1}-\left(1-\frac{9}{4} s^{2}\right) u_{1}^{2}\right] \mathrm{d} w_{1}=0 .
\end{aligned}
$$

The expression for $\dot{w}_{1}^{2}$ is extracted from Eq. (21) and the result is substituted into Eq. (20). Then the following equation is derived:

$$
\begin{aligned}
& u_{1}^{\prime \prime} p^{2}\left\{s^{2}\left(1+\frac{3}{4} s^{2}\right)\left(w_{1}^{2}-W_{1}^{(\max )^{2}}\right)+\left(\frac{1}{2}+\frac{3}{4} s^{2}\right)\left(W_{1}^{(\max )^{4}}-w_{1}^{4}\right)\right\} \\
& \quad+2 u_{1}^{\prime \prime} p^{2} \int_{W_{1}^{(\max )}}^{w_{1}} w_{1}\left[c\left(1+\frac{3}{2} s^{2}\right) u_{1}+\left(1-\frac{9}{4} s^{2}\right) u_{1}^{2}\right] \mathrm{d} w_{1}-p^{2}\left[-s^{2}\left(1+\frac{3}{4} s^{2}\right) w_{1}\right. \\
& \left.\quad-c\left(1+\frac{3}{2} s^{2}\right) w_{1} u_{1}-\left(1-\frac{9}{4} s^{2}\right) u_{1}^{2} w_{1}+\left(1+\frac{3}{2} s^{2}\right) w_{1}^{3}\right] u_{1}^{\prime}+u_{1}=\varepsilon \bar{\gamma}\left\{-u_{1}+\frac{15}{4} c s^{2} u_{1}^{2}\right. \\
& \left.\quad+c\left(\frac{1}{2}+\frac{3}{4} s^{2}\right) w_{1}^{2}+\left(1-\frac{9}{4} s^{2}\right) u_{1} w_{1}^{2}+\left(\frac{5}{2}-\frac{15}{4} s^{2}\right) u_{1}^{3}\right\}+\varepsilon f g\left(w_{1}\right) .
\end{aligned}
$$

A trajectory of the NNM in the form (19) is substituted into Eq. (22) and the dominant terms by $\varepsilon$ are retained. As a result the asymptotic equation of the first approximation with respect to $\varepsilon$ is derived in the following form:

$$
\begin{aligned}
& \tilde{u}^{\prime \prime} p^{2}\left\{s^{2}\left(1+\frac{3}{4} s^{2}\right)\left(w_{1}^{2}-W_{1}^{(\max )^{2}}\right)+\left(\frac{1}{2}+\frac{3}{4} s^{2}\right)\left(W_{1}^{(\max )^{4}}-w_{1}^{4}\right)\right\} \\
& \quad-p^{2}\left[-s^{2}\left(1+\frac{3}{4} s^{2}\right) w_{1}+\left(1+\frac{3}{2} s^{2}\right) w_{1}^{3}\right] \tilde{u}^{\prime}+\tilde{u}=\bar{\gamma} c\left(\frac{1}{2}+\frac{3}{4} s^{2}\right) w_{1}^{2}+f g\left(w_{1}\right)+O(\varepsilon) .
\end{aligned}
$$

Two boundary conditions at $w_{1}= \pm W_{1}^{(\max )}$, which guarantee an analytical continuation of the trajectory up to the maximum equipotential surface $[21,22]$, are derived from $(23)$

$$
\begin{aligned}
- & p^{2}\left[\mp s^{2}\left(1+\frac{3}{4} s^{2}\right) W_{1}^{(\max )} \pm\left(1+\frac{3}{2} s^{2}\right) W_{1}^{(\max )^{3}}\right] \tilde{u}^{\prime}\left( \pm W_{1}^{(\max )}\right)+\tilde{u}\left( \pm W_{1}^{(\max )}\right) \\
& =\bar{\gamma} c\left(\frac{1}{2}+\frac{3}{4} s^{2}\right) W_{1}^{(\max )^{2}}+f g\left( \pm W_{1}^{(\max )}\right) .
\end{aligned}
$$

The following series is obtained from Eqs. (10) and (19):

$$
\tilde{u}=a_{0}+a_{1} W_{1}^{(\max )} \cos \varphi+a_{2} W_{1}^{(\max )^{2}} \cos ^{2} \varphi+\cdots .
$$


Series (25) is substituted into Eq. (23) and the coefficients of $\cos ^{0} \varphi, \cos ^{1} \varphi, \cos ^{2} \varphi$ are equated. As a result three linear algebraic equations with respect to five variables $a_{0}, \ldots, a_{4}$ are derived. Two additional linear algebraic equations are obtained from boundary conditions (24). Thus the system of five linear algebraic equations is obtained. The solution of this system is the following:

$$
\begin{gathered}
a_{0}=-\frac{\bar{\mu} c L_{3} L_{4}}{36\left[\left(4 s^{2}+p^{-2}\right) L_{3}+L_{4}\right]}, \quad a_{1}=f \frac{L_{2}}{L_{1}}, \\
a_{2}=\bar{\mu} c \frac{\left(2+3 s^{2}\right) L_{3}}{4\left[\left(4 s^{2}+p^{-2}\right) L_{3}+L_{4}\right]}, \quad a_{3}=\frac{f}{L_{5}}\left[A_{1}+A_{3}-\frac{L_{2}}{L_{1}}\left(W_{1}^{(\max )}+p^{2} \chi\right)\right], \\
a_{4}=\frac{3 \bar{\gamma} c\left(1+3 s^{2}\right)}{2\left[\left(4 s^{2}+p^{-2}\right) L_{3}+L_{4}\right]},
\end{gathered}
$$

where

$$
\begin{gathered}
L_{1}=\left(1+7 p^{2} s^{2}\right) W_{1}^{(\max )}+3 \chi p^{2}\left(1+3 p^{2} s^{2}\right)-6 p^{2} W_{1}^{(\max )^{2}}\left(W_{1}^{(\max )}+p^{2} \chi\right)\left(\frac{1}{2}+\frac{3}{4} s^{2}\right), \\
\left.L_{2}=\left(A_{1}-3 A_{3}\right) \quad 1+\frac{3 \chi p^{2}}{W_{1}^{(\max )}}\right)-6 p^{2}\left(A_{1}+A_{3}\right)\left[-s^{2}+\left(\frac{1}{2}+\frac{3}{4} s^{2}\right) W_{1}^{(\max )^{2}}\right] \\
L_{3}=1+16 p^{2} s^{2}-p^{2} W_{1}^{(\max )^{2}}\left(10+15 s^{2}\right), \\
L_{4}=12 p^{2} W_{1}^{(\max )^{2}}\left(3+\frac{9}{2} s^{2}\right)\left[\left(\frac{1}{2}+\frac{3}{4} s^{2}\right) W_{1}^{(\max )^{2}}-s^{2}\right] \\
L_{5}=W_{1}^{(\max )^{3}}+p^{2} \chi^{3} W_{1}^{(\max )^{2}}, \quad \chi=s^{2} W_{1}^{(\max )}-\left(1+\frac{3}{2} s^{2}\right) W_{1}^{(\max )^{3}} .
\end{gathered}
$$

Now nonlinear normal vibrations mode (19) and (26) is considered for the following system parameters: $\gamma=\mu=\varepsilon=0.01 ; \varphi=0.15 ; f=0.05 ; \omega=0.2033 ; W_{1}^{(\max )}=0.3$. Fig. 4a shows the NNM trajectory in the configuration space. We can see that this motion has significant amplitudes of the snap-through absorber and small amplitudes of the main linear subsystem.

Numerical simulations of System (5) were carried out to compare the analytical results and simulations at the different values of the system parameters. System (5) is integrated by the Runge-Kutta method. The initial conditions of NNM (19) are taken for the calculations:

$$
u_{1}(0)=\varepsilon \tilde{u}\left(W_{1}^{(\max )}\right), \quad \dot{u}_{1}(0)=0, \quad w_{1}(0)=W_{1}^{(\max )}, \quad \dot{w}_{1}(0)=0 .
$$

Fig. $4 \mathrm{~b}$ shows the results of the numerical integration in the configuration space. Figs. 5 and 6 show the nonlinear normal modes obtained analytically and numerically at $\omega=0.304595$ and 0.333166 . Fig. $7 \mathrm{a}$ and $\mathrm{b}$ show the time histories of the nonlinear normal vibrations mode obtained analytically and numerically at $\omega=0.333166$. As follows from the calculations, the analytical results are close to the data of the simulations. Simulations (Figs. 4b, 5b, 6b) do not show a simple nonlinear modal curve, but show the quasi-periodic trajectory which is generally near the predicted nonlinear mode curve. It appears that this is due to the fact that the predicted initial conditions are only approximate, and lead to quasi-periodic response, whereas if one knew the 


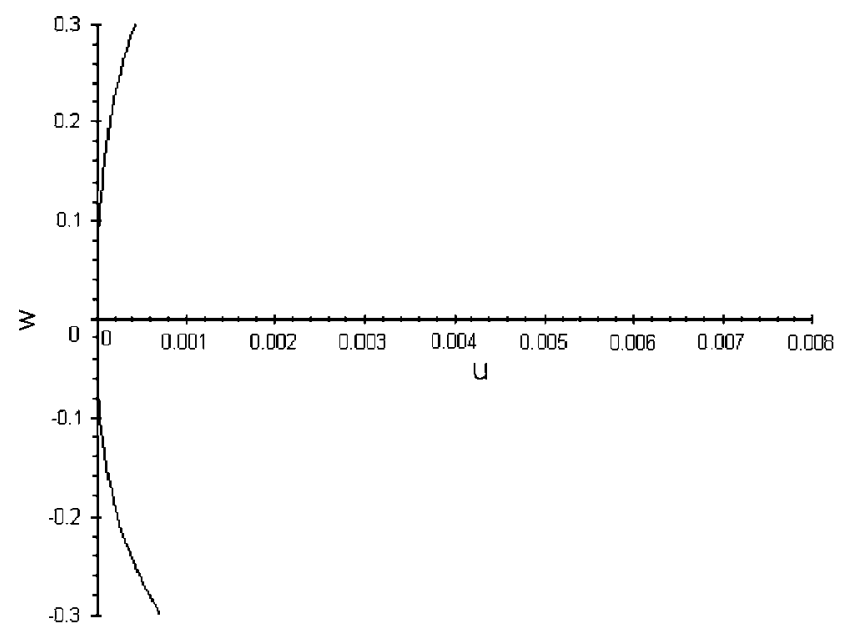

(a)

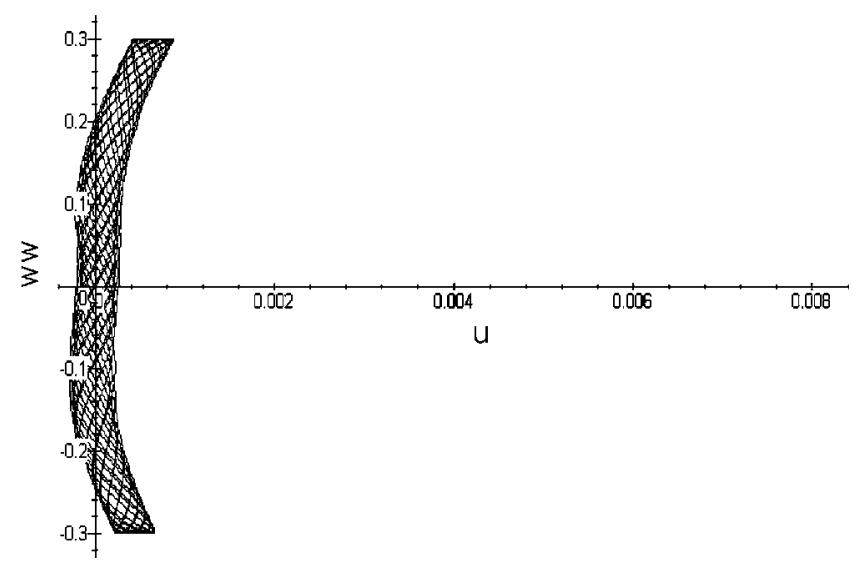

(b)

Fig. 4. The forced nonlinear normal vibrations mode at $\omega=0.2033 ; W_{1}^{(\max )}=0.3$ : (a) the nonlinear normal vibration mode, which is calculated by formulae (19) and (26); (b) results of the numerical calculation.

nonlinear modal initial conditions more precisely, the normal mode curve would be followed more exactly.

The analytical results are used to analyze the frequency response, which is the dependence of the amplitude of the first harmonics on $\omega$ :

$$
A_{\Sigma}(\omega)=\varepsilon\left(a_{1} W_{1}^{(\max )}+\frac{3}{4} a_{3} W_{1}^{(\max )^{3}}\right) .
$$

Fig. 8 shows the frequency response, which has two resonance regions.

Nonlinear normal vibrations mode (19) has small amplitudes of $u_{1}: u_{1}=O(\varepsilon)$. Therefore, the first approximation with respect to $\varepsilon$ of Eq. (5a) is linear and the frequency response is similar to a 


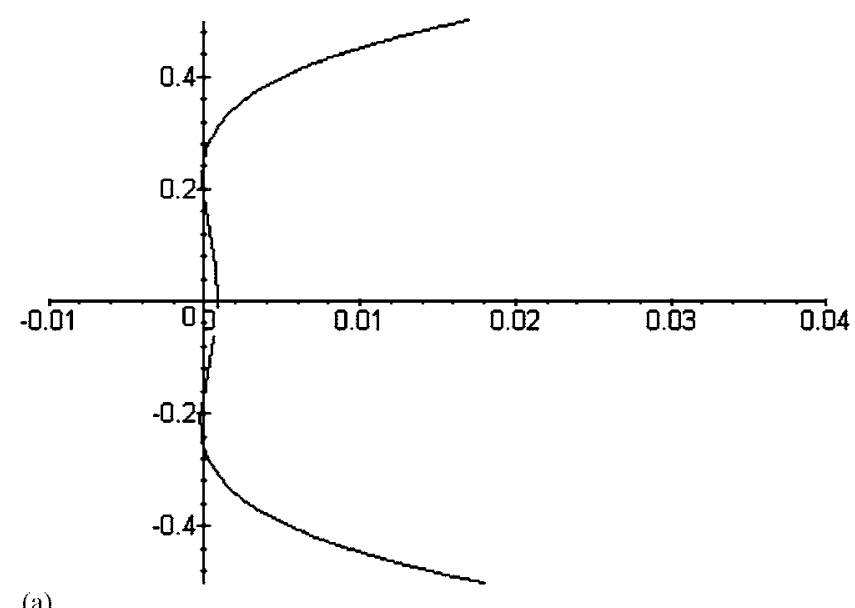

(a)

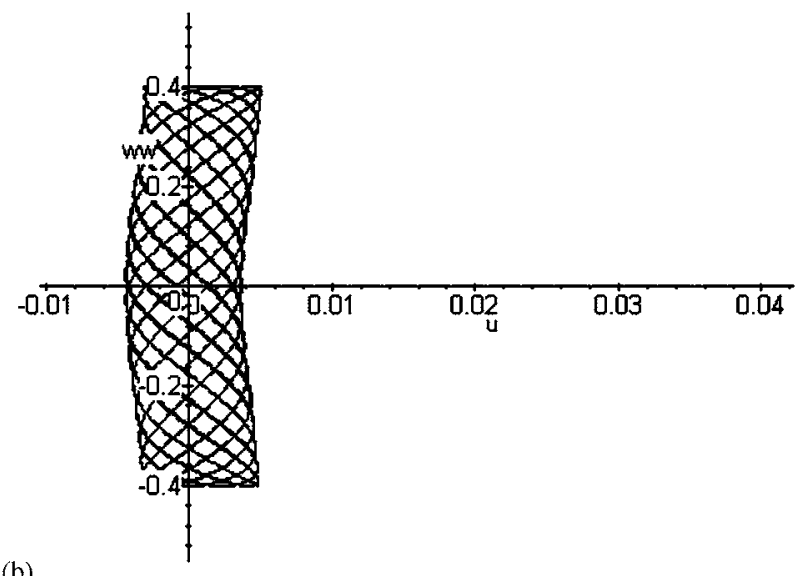

(b)

Fig. 5. The forced nonlinear normal vibrations mode at $\omega=0.304595 ; W_{1}^{(\max )}=0.4$ : (a) the nonlinear normal vibration mode, which is calculated by formulae (19) and (26): (b) results of the numerical calculation.

linear one. The first approximation of Eq. (5b) describes free oscillations of the snapthrough truss. Fig. 9 shows the backbone curve of these oscillations. The backbone curve starts at a non-zero point since it corresponds to the snap-through motions only, which begin at non-zero amplitudes. The second approximation of frequency response (28) has bending. However, the first approximation is enough to study a principal possibility of vibrations absorption.

Due to nonlinearity of the snap-through absorber, two resonances corresponding to the nonlinear normal vibrations mode (19) take place in system (5) (see Fig. 8). To treat this conclusion let us consider the main subsystem with linear absorber (Fig. 10). Then only one resonance of motions $u_{1}=\varepsilon \tilde{u}\left(w_{1}\right)$ takes place. The second resonance in the two-dof linear system has motions, which essentially differ from the mode $u_{1}=\varepsilon \tilde{u}\left(w_{1}\right)$. 


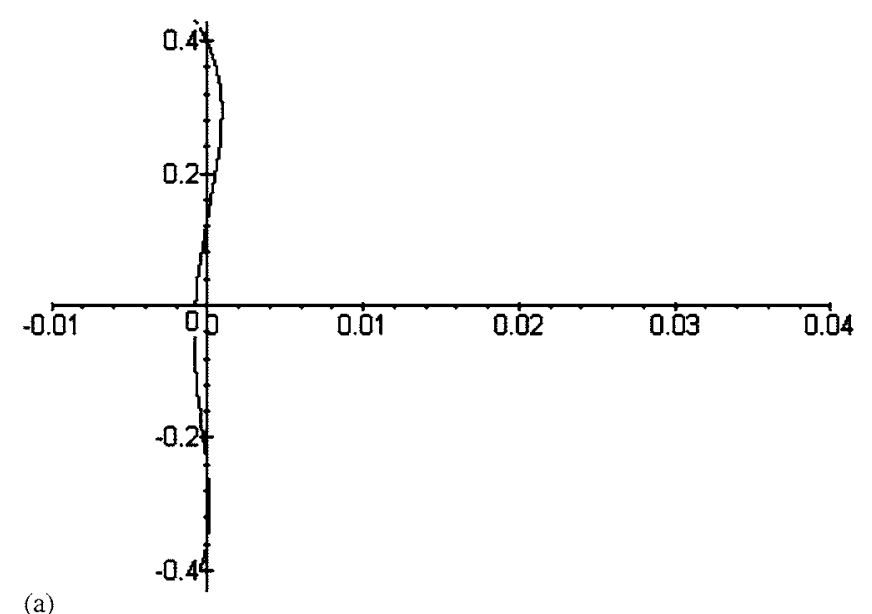

(a)

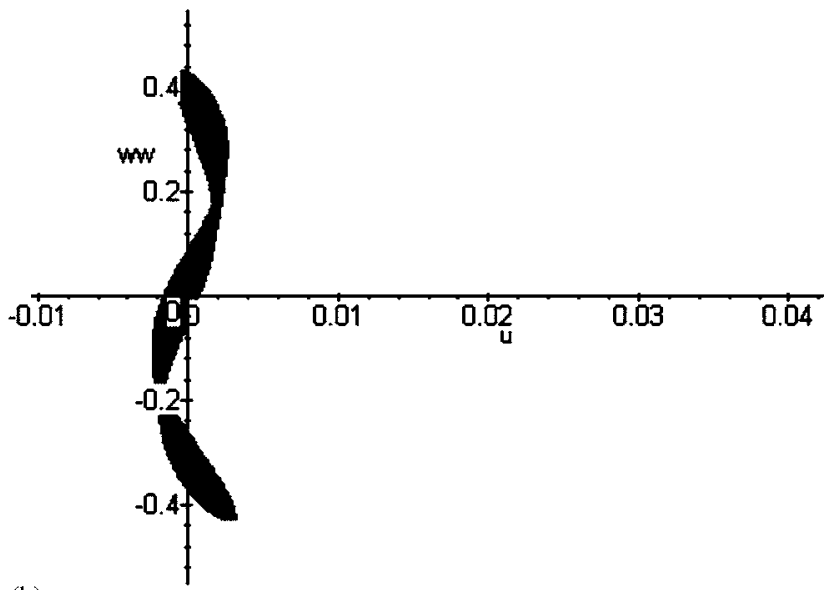

(b)

Fig. 6. The forced nonlinear normal vibrations mode at $\omega=0.333166 ; W_{1}^{(\max )}=0.43$ : (a) the nonlinear normal vibration mode, which is calculated by formulae (19) and (26); (b) results of the numerical calculation.

The locations of the resonances of the nonlinear system (Fig. 1) make possible to absorb the dangerous oscillations of the main linear subsystem. Moreover, the advantage of the snap-through absorber is that the vibrations absorption mode exists in a wide frequency range.

Now the results of numerical analysis of the vibrations absorption mode in damped system (2) are presented. System (2) is integrated with the above-presented parameters and $\beta_{1}=0.1 ; \beta_{2}=0.001 ; f=1 ; \omega=0.65$. The initial conditions are taken from the obtained analytically vibrations absorption mode (26) and (19). Fig. 11 shows the results of the numerical simulations, which demonstrate the existence of the vibration absorption mode in the damped system when the amplitudes of vibrations of the linear subsystems are small and the snap-through absorber jumps through three equilibrium positions. 


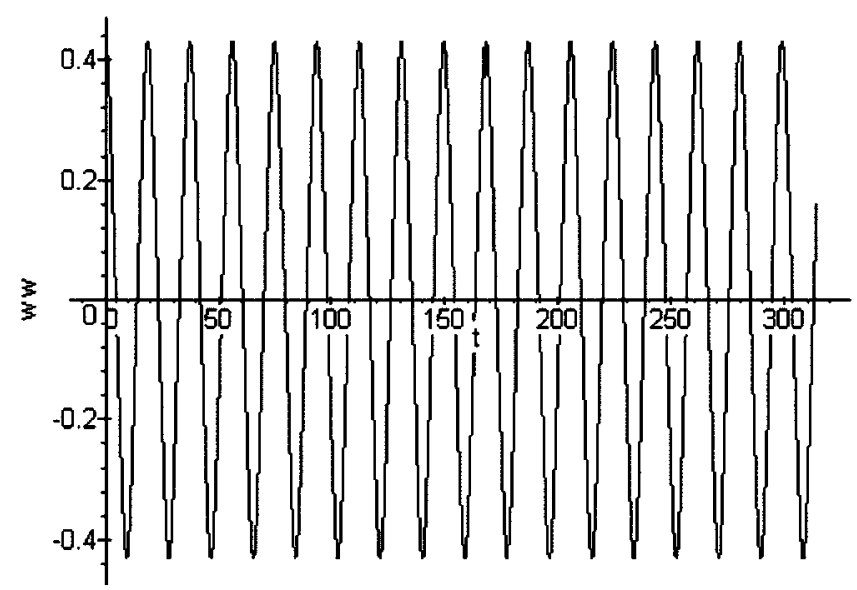

(a)

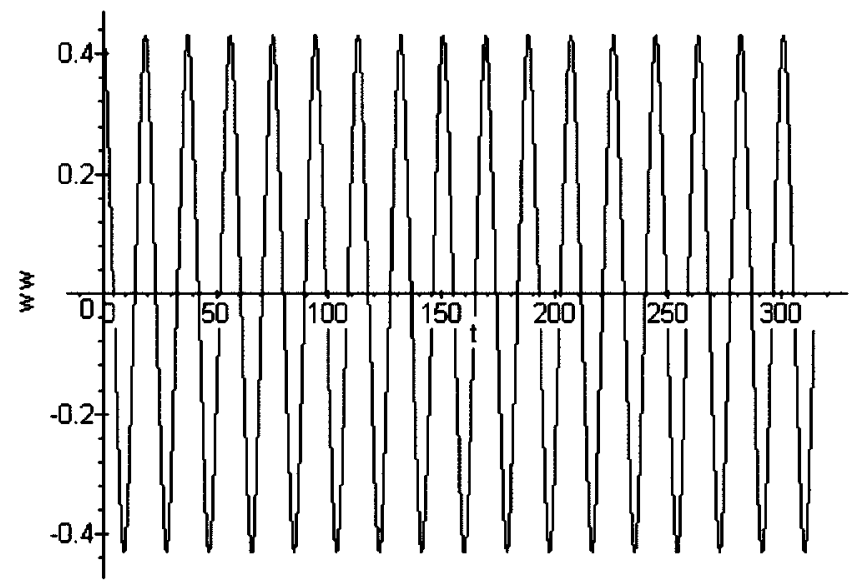

(b)

Fig. 7. Time history of oscillations at $\omega=0.333166$ : (a) analytical results; (b) data of numerical simulations of system (5).

Let us compare the efficiency of the linear and the snap-through absorber. One considers horizontal vibrations of the two-dof system, which consists of the single-dof linear oscillator with a big mass and a linear absorber attached to this mass (Fig. 10). External periodic force $\varepsilon f \cos (\omega t)$ acts on big mass $M$. The stiffness of the spring and mass of the linear absorber are equal to the corresponding parameters of the snap-through truss. The system parameters are taken dimensionless: $c_{1}=1 ; M=1 ; \tilde{c}=\varepsilon \bar{\gamma} ; m=\varepsilon \bar{\mu}$. The oscillations amplitudes of the main mass $a_{1}$ can be determined in the following form [1]:

$$
a_{1}=\varepsilon f \frac{1-\left(\omega^{2} / \omega_{a}^{2}\right)}{\left(1-\left(\omega^{2} / \omega_{a}^{2}\right)\right)\left(1+\varepsilon \bar{\gamma}-\omega^{2}\right)+\varepsilon \bar{\gamma}},
$$

where $\omega_{a}^{2}=\bar{\gamma} / \bar{\mu}$. Fig. 12 shows the frequency response $a_{1}(\omega)$. Thus, in the system with the linear absorber the resonances take place at $\omega \approx 0.92$ and 1.04 (Fig. 12). These frequencies are close to 


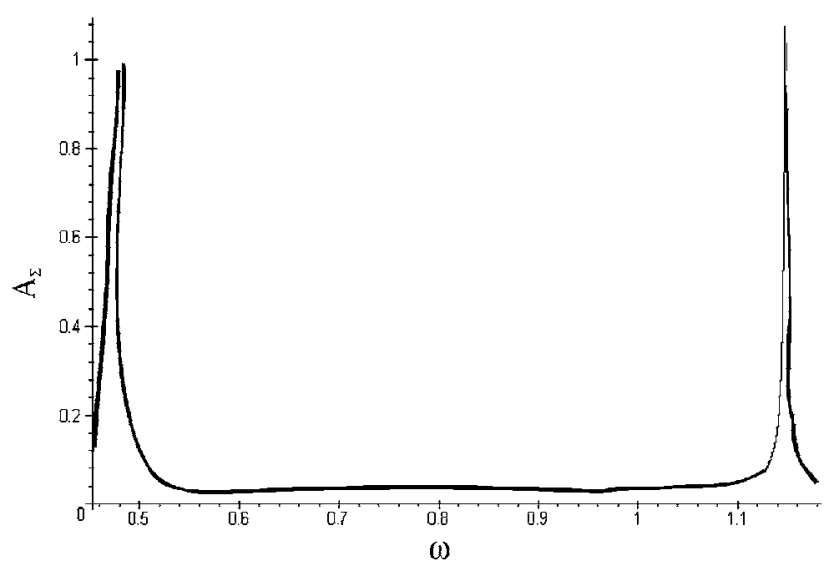

Fig. 8. The frequency response of the main linear subsystem forced oscillations.

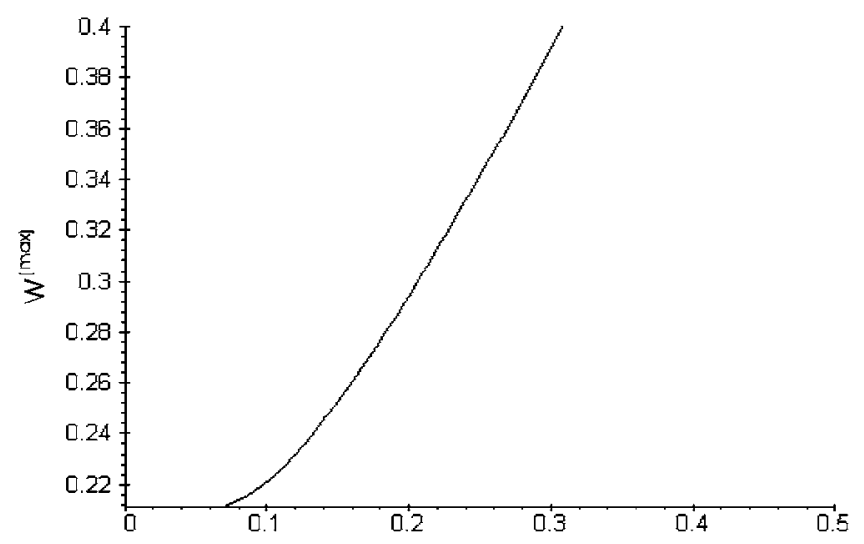

Fig. 9. The backbone curve of the snap-through truss oscillations.

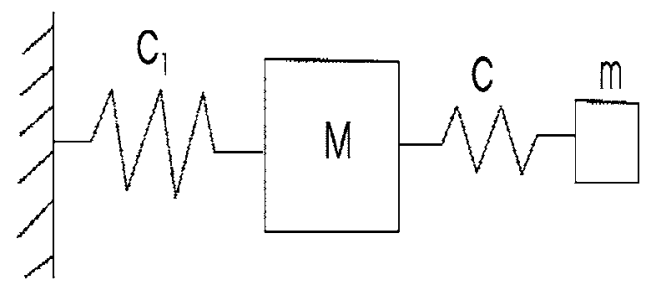

Fig. 10. The system with linear absorber.

$\omega=1$, when the resonance in the main system without absorber occurs. Therefore, the linear absorber with the considered parameters is not effective. We stress that for the chosen parameters there are not resonances in the system with the snap-through absorber at frequency range $\omega \in[0.55 ; 1.1]$. Therefore, the snap-through absorber is effective. 

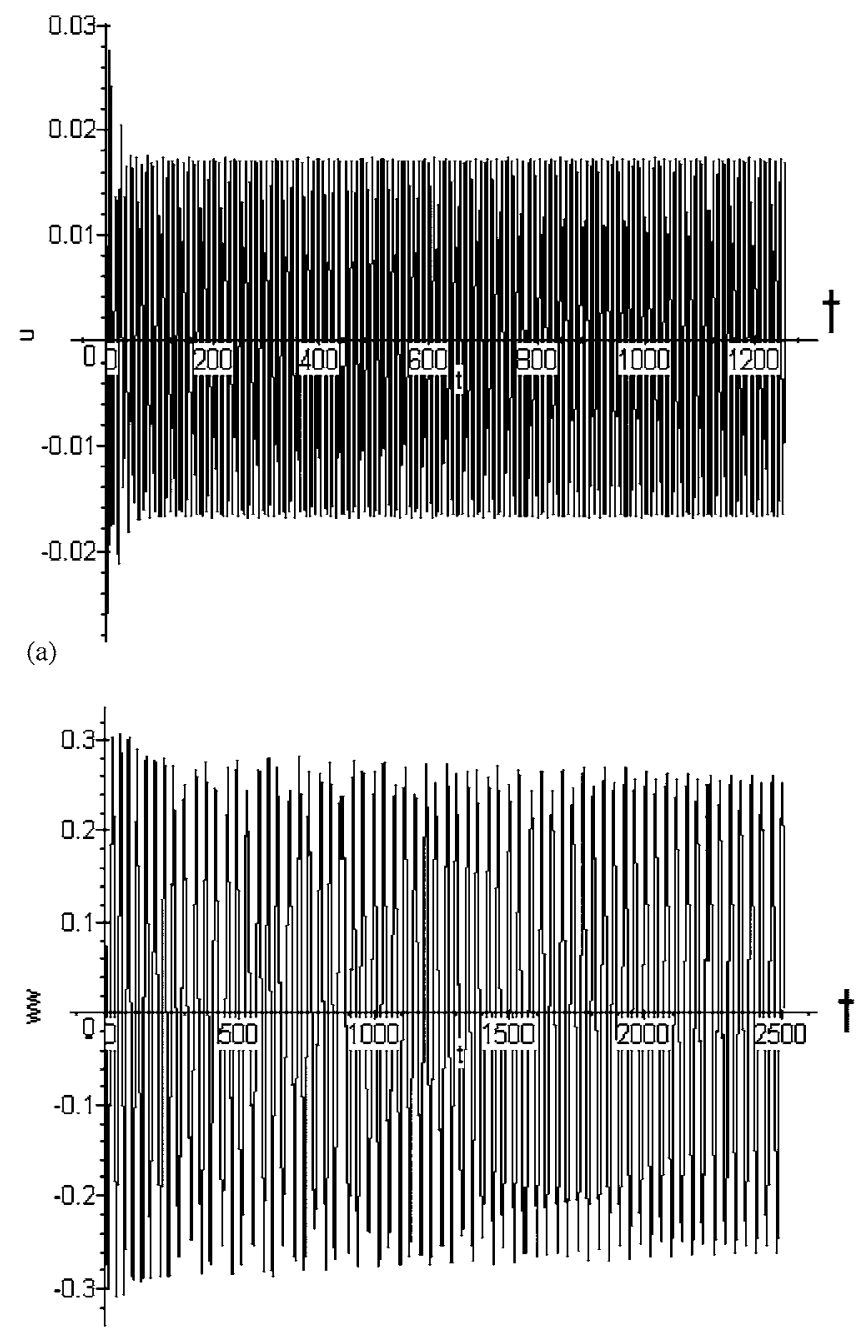

(b)

Fig. 11. The time history of vibration absorption mode of the damped system: (a) the time history of the general coordinate of the main linear subsystem; (b) the time history of the general coordinate of the snap-through absorber.

\section{Stability of the vibrations absorption mode}

Expression (19) is substituted into system (5) to study a stability of nonlinear normal vibrations mode. Then the following system is obtained:

$$
\begin{gathered}
\ddot{\tilde{u}}+\tilde{u}=\bar{\gamma} c\left(\frac{1}{2}+\frac{3}{4} s^{2}\right) w_{1}^{2}+f \cos (\omega \tau)+\varepsilon \bar{\gamma}\left[-\tilde{u}+\left(1-\frac{9}{4} s^{2}\right) \tilde{u} w_{1}^{2}\right]+O\left(\varepsilon^{2}\right), \\
\ddot{w}_{1}+p^{2} w_{1}\left[-s^{2}\left(1+\frac{3}{4} s^{2}\right)+\left(1+\frac{3}{2} s^{2}\right) w_{1}^{2}\right]+O(\varepsilon)=0 .
\end{gathered}
$$




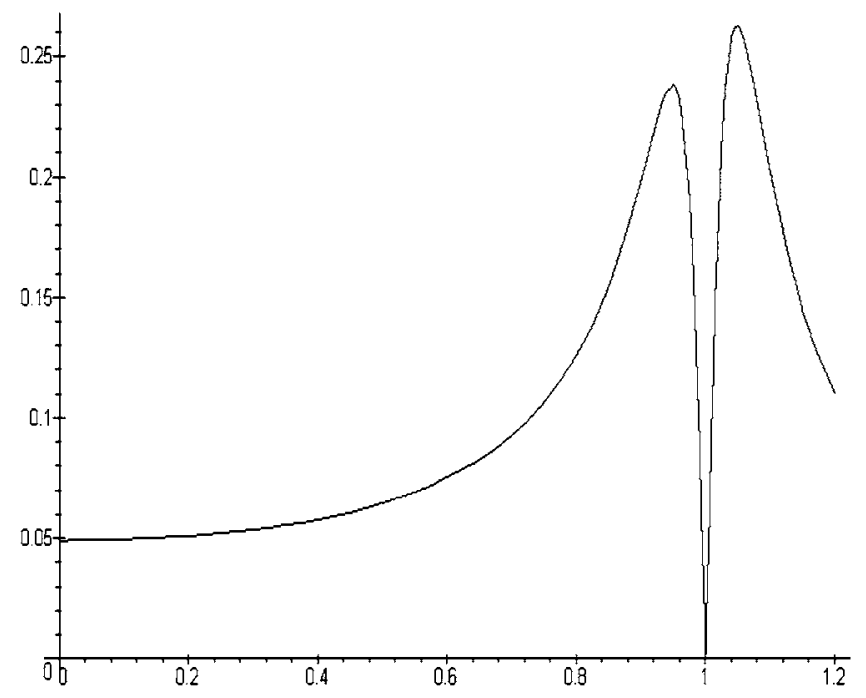

Fig. 12. The frequency response of the system with linear absorber.

Eq. (30b) is $u_{1}$-independent within the terms of order $\varepsilon$ and Eq. (30b) has analytical solutions (10). To study stability of periodic solutions, Eq. (30a) is considered. Then the equation with respect to small deviations $\xi(t)$ from the periodic motions is derived in the following form:

$$
\ddot{\xi}+\xi\left[1+\varepsilon \bar{\gamma}-\varepsilon \bar{\gamma} w_{1}^{2}(t)\right]=0 .
$$

Using the Fourier series for $c n^{2}(2 K \omega t / \pi)$, Eq. (31) can be written as

$$
\ddot{\xi}+\xi\left[1-\varepsilon \Omega_{1}-\varepsilon \chi \sum_{v=1}^{\infty} \frac{v q^{v}}{1-q^{2 v}} \cos (2 v \omega t)\right]=0,
$$

where

$$
\begin{gathered}
\chi=\bar{\gamma} W_{1}^{(\max )^{2}} 2 \pi^{2} K^{-2} k^{-2}, \quad q=\exp \left(-\frac{\pi K^{\prime}}{K}\right), \\
\Omega_{1}=\bar{\gamma}\left[W_{1}^{(\max )^{2}} K^{-1} k^{-2}\left(E-k^{\prime 2} K\right)-1\right] .
\end{gathered}
$$

Let us consider the set of parametric resonances:

$$
\omega=\frac{1}{r}+\varepsilon \frac{\sigma}{r}, \quad r=1,2, \ldots,
$$

where $\sigma$ is the detuning parameter. The multiple scales method is used to analyze Eq. (32). Solutions of this equation are presented as

$$
\xi=\xi_{0}\left(T_{0}, T_{1}, \ldots\right)+\varepsilon \xi_{1}\left(T_{0}, T_{1}, \ldots\right)+\cdots,
$$


where $T_{0}=t ; T_{1}=\varepsilon t \ldots$. Expansion (34) is substituted into Eq. (32) and the terms of the same orders of $\varepsilon$ are collected. As a result, the next equations are derived:

$$
\begin{gathered}
\xi_{0}=A\left(T_{1}\right) \exp \left(\mathrm{i} T_{0}\right)+\bar{A}\left(T_{1}\right) \exp \left(-\mathrm{i} T_{0}\right), \\
\frac{\partial^{2} \xi_{1}}{\partial T_{0}^{2}}+\xi_{1}=-2 \frac{\partial^{2} \xi_{0}}{\partial T_{0} \partial T_{1}}+\Omega_{1} \xi_{0}+\chi \xi_{0} \sum_{v=1}^{\infty} \frac{v q^{v}}{1-q^{2 v}} \cos (2 v \omega t) .
\end{gathered}
$$

Excluding the secular terms from Eq. (35) and using the change of the variables $A=(a / 2) \exp (\mathrm{i} \psi)$, the following system of modulation equations is obtained:

$$
a^{\prime}=\tilde{g}_{r} a \sin \left(2 \sigma T_{1}-2 \psi\right), \quad \psi^{\prime}=-\frac{\Omega_{1}}{2}-\tilde{g}_{r} \cos \left(2 \sigma T_{1}-2 \psi\right),
$$

where $\tilde{g}_{r}=\chi q^{r} r /\left(2\left(1-q^{2 r}\right)\right)$. System (36) is rewritten with respect to Cartesian coordinates $(x, y)=a\left\lfloor\cos \left(\sigma T_{1}-\psi\right), \sin \left(\sigma T_{1}-\psi\right)\right\rfloor$ :

$$
x^{\prime}=\left[\tilde{g}_{r}-\frac{\Omega_{1}}{2}-\sigma\right] y, \quad y^{\prime}=\left[\tilde{g}_{r}+\frac{\Omega_{1}}{2}+\sigma\right] x .
$$

As follows from (37), boundaries of unstable regions are described by two equations:

$$
\tilde{g}_{r}=\frac{\Omega_{1}}{2}+\sigma, \quad \tilde{g}_{r}=-\frac{\Omega_{1}}{2}-\sigma .
$$

Two values $\sigma_{1,2}$, which are derived from Eqs. (38) and (33), determine the boundaries of the unstable oscillations on the frequency response (Fig. 8). These values can be calculated from the equations:

$$
\sigma_{1,2}= \pm \frac{2 \bar{\mu}}{r \operatorname{sh}\left(r \pi K^{\prime} / K\right)}+\frac{\bar{\gamma}}{2}\left(-\frac{4 \sqrt{W_{1}^{(\max )^{2}}-s^{2} E}}{r \pi p}+W_{1}^{(\max )^{2}}+1\right) .
$$

Note that the next equation connects $\xi$ to the variables of system (37):

$$
\xi=x \cos (r \omega t)+y \sin (r \omega t)+O(\varepsilon) .
$$

Let us consider the instability region close to the first resonance $(r=1 ; \omega \approx 1)$. In this case system (5) oscillations with period $T=2 \pi / \omega$ become unstable and the new motions of the same order arise. Now the numerical analysis of this resonance region is performed. Let us take the system parameters from the previous section and $\omega \approx 1, W_{1}^{(\max )}=1.185$. Then the unstable oscillations are observed in the frequency range $\omega \in[1 ; 1.0003]$. It is important that this range is very narrow. The other unstable regions $(33)(r=2,3, \ldots)$ are smaller than the first one, as the amplitudes of the higher harmonics in Eq. (32) are decreased when the index $v$ is increased.

\section{Conclusion}

It is determined in this paper that in the two-dof discrete system with the snap-through absorber, the absorption mode exists in the form of the nonlinear normal vibrations mode. Using the combination of the nonlinear normal vibrations mode approach, the Rauscher method and 
asymptotic analysis, the absorption mode is derived analytically. The numerical results presented here verify the reliability of the analytical analysis. It is established that significant oscillations amplitudes of the main mass in the system with the snap-through absorber are not observed in the resonance frequency range of the main linear subsystem without the absorber. The stability of the vibration absorption mode is considered. The analysis shows that the vibration absorption mode is stable.

The snap-through absorber is compared with the linear one. The mass and stiffness of the linear absorber are suggested to be equal to the corresponding parameters of the snap-through truss. The calculations show that resonance oscillations in the main subsystem are not successful in absorbing by means of the linear absorber, but the snap-through absorber leads to small oscillations amplitudes of the main subsystem in the wide frequency range. Thus, the advantage of the snap-through absorber is that it works over a wide frequency range.

\section{Acknowledgements}

The authors especially thank Profs. O. Gendelman, A.I. Manevich, A. Vakakis, F. Pellicano for fruitful discussions on the subject of this paper.

\section{References}

[1] J.P. Den Hartog, Mechanical Vibrations, McGraw-Hill, New on Dover, 1985.

[2] A.H. Nayfeh, D.T. Mook, Nonlinear Oscillations, Wiley-Interscience, New York, 1979.

[3] V.D. Kubenko, P.S. Kovalchuk, T.S. Krasnopol'skaya, Nonlinear Interactions of Bending Oscillations Modes in Cylindrical Shells, Naukova Dumka, Kiev, 1984 (in Russian).

[4] R.S. Haxton, A.D.S. Barr, The autoparametric vibration absorber, Journal of Engineering for Industry 94 (1972) 119-222.

[5] S.W. Shaw, S. Wiggins, Chaotic motions of a torsional vibration absorber, Transactions of the ASME. Journal of Applied Mechanics 55 (1988) 952-958.

[6] J. Shaw, S.W. Shaw, A.G. Haddow, On the response of the non-linear vibration absorber, International Journal of Non-Linear Mechanics 24 (4) (1989) 281-293.

[7] S. Natsiavas, Steady state oscillations and stability of non-linear dynamic vibration absorber, Journal of Sound and Vibration 156 (22) (1992) 227-245.

[8] S. Natsiavas, Vibration absorbers for a class of self-excited mechanical systems, Transactions of the ASME. Journal of Applied Mechanics 60 (1993) 382-387.

[9] C.-T. Lee, S.W. Shaw, Torsional vibration reduction in internal combustion engines using centrifugal pendulums, in: Proceedings of the Design Engineering Technical Conference, Vol. 3(A), ASME, USA, 1995, pp. 487-492.

[10] A.G. Haddow, S.W. Shaw, An experimental study of torsional vibration absorber, in: Proceedings of the ASME Design Engineering Technical Conference, USA, 2001, pp. 1-7.

[11] A. Vakakis, Inducing passive nonlinear energy sinks in vibrating system, Transactions of the ASME. Journal of Vibration and Acoustics 123 (2001) 324-332.

[12] A. Vakakis, An overview of motion localization and energy pumping in coupled nonlinear oscillators, in: Proceedings of Sixth Conference on Dynamical Systems Theory and Application, Lodz, Poland, 2001, pp. 111-118.

[13] A. Vyas, A.K. Bajaj, Dynamics of autoparametric vibration absorbers using multiple pendulums, Journal of Sound and Vibration 246 (1) (2001) 115-135.

[14] M.P. Karyeaclis, T.K. Caughey, Stability of a semi-active impact damper: Part I-global behavior, Transactions of the ASME. Journal of Applied Mechanics 56 (1989) 926-929. 
[15] M.P. Karyeaclis, T.K. Caughey, Stability of a semi-active impact damper: Part II—periodic solution, Transactions of the ASME. Journal of Applied Mechanics 56 (1989) 930-940.

[16] S. Aoki, T. Watanabe, An investigation of an impact vibration absorber with hysteretic damping, in: Proceedings of the Second International Conference on Motion and Vibration Control, Yokohama, Japan, 1994, pp. 468-474.

[17] S. Shaw, S.W. Shaw, The onset of chaos in a two-degree-of-freedom impacting system, Transaction of the ASME. Journal of Applied Mechanics 56 (1989) 168-174.

[18] K.V. Avramov, Yu.V. Mikhlin, Snap-through truss as a vibration absorber, Journal of Vibration and Control 10 (2) (2004) 291-308.

[19] K.V. Avramov, Yu.V. Mikhlin, Forced oscillations of system, containing snap-through truss, close to equilibrium position, Nonlinear Dynamics 35 (2004) 361-379.

[20] G.V. Manucharyan. Construction of homoclinic trajectories in forced and parametrically excited nonlinear systems, in: G.S. Osipenko (Ed.), Proceedings of Fourth International Conference. Tools for Mathematical Modeling, Sankt-Petersburg, 2003, pp. 253-263.

[21] A.F. Vakakis, L.I. Manevitch, Yu.V. Mikhlin, V.N. Pilipchuk, A.A. Zevin, Normal Modes and Localization in Nonlinear Systems, Wiley-Interscience, New York, 1996.

[22] L.I. Manevitch, Yu.V. Mikhlin, V.N. Pilipchuk, Method of Normal Modes for Essentially Nonlinear Systems, Nauka, Moscow, 1989 (in Russian).

[23] J. Guckenheimer, P. Holmes, Nonlinear Oscillations, Dynamical Systems, and Bifurcations of Vector Fields, Springer, New York, 1984.

[24] P. Byrd, M. Friedman, Handbook of Elliptic Integrals for Engineers and Scientists, Springer, Berlin, 1954. 